\title{
Chemical Fingerprinting and Antimicrobial Evaluation of the Methanolic Extract of the Leaves of the Endemic Cuban Plant Coccoloba cowellii ${ }^{+}$
}

\author{
Daniel Méndez ${ }^{1}$, Julio C. Escalona ${ }^{2}$, Luc Pieters ${ }^{3}$ and Paul Cos ${ }^{4, *}$
}

Citation: Méndez, D.; Escalona, J.C.; Pieters, L.; Cos, P. Chemical Fingerprinting and Antimicrobial Evaluation of the Methanolic Extract of the Leaves of the Endemic Cuban Plant Coccoloba cowellii. Chem. Proc. 2021, 3, 124. https://doi.org/10.3390/ ecsoc-24-08289

Academic Editors: Julio A. Seijas and M. Pilar Vázquez-Tato

Published: 14 November 2020

Publisher's Note: MDPI stays neutral with regard to jurisdictional claims in published maps and institutional affiliations.

Copyright: $@ 2020$ by the authors. Licensee MDPI, Basel, Switzerland. This article is an open access article distributed under the terms and conditions of the Creative Commons Attribution (CC BY) license (http://creativecommons.org/licenses/by/4.0/).
1 Chemistry Department, Faculty of Applied Sciences, University of Camagüey, Carretera de Circunvalación Km 5 1⁄2, Camagüey 74650, Cuba; daniel.mendez@reduc.edu.cu

2 Pharmacy Department, Faculty of Natural and Exact Sciences, Oriente University, Avenida Patricio Lumumba s/n, Santiago de Cuba 90500, Cuba; jcea@uo.edu.cu

3 Natural Products \& Food Research and Analysis (NatuRA), Department of Pharmaceutical Sciences, University of Antwerp, Universiteitsplein 1, B-2610 Antwerp, Belgium; luc.pieters@uantwerpen.be

4 Laboratory of Microbiology, Parasitology and Hygiene (LMPH), Faculty of Pharmaceutical, Biomedical and Veterinary Sciences, University of Antwerp, Universiteitsplein 1, B-2610 Antwerp, Belgium

* Correspondence: paul.cos@uantwerpen.be

+ Presented at the 24th International Electronic Conference on Synthetic Organic Chemistry, 15 November-15 December 2020; Available online: https://ecsoc-24.sciforum.net/.

\begin{abstract}
The genus Coccoloba (Polygonaceae) comprises approximately 150 species of flowering plants. It is native to the tropical and subtropical regions of America, in South America, the Caribbean and Central America. A wide variety of biological activities has been studied for Coccoloba species due to great diversity of metabolites (mainly flavonoids, tannins, terpenoids and volatile oils) and popular uses reported for different ailments like fever, diarrhea, menstrual disturbance, uterine hemorrhages, hemorrhoids and gonorrhea. The methanolic extract of the leaves of C. cowellii, endemic of the Camagüey province of Cuba and critically endangered, was subjected to structural analysis. The obtained HPLC-DAD-QTOF-ESI-MS data were analyzed employing the MSDIAL software. A dereplication of the ESI-MS data was realized using the feature-based molecular networking (FBMN) analysis method in the Global Natural Products Social Molecular Networking (GNPS) infrastructure, leading to 12 compound hits against the GNPS database. A total of 13 compounds were tentatively identified by means of MS data, together with the interpretation of the observed MS/MS spectra in comparison with those found in the literature. The major compounds were myricetin and quercetin glucuronides and glycosides and epichatechin-3-O-gallate. The total extract presented an antifungal effect against Candida albicans ATCC B59630 (azole-resistant) (IC50 $=2.13 \mu \mathrm{g} / \mathrm{mL}$ ) and was not cytotoxic (IC50 > 64.00 $\mu \mathrm{g} / \mathrm{mL}$ ) in the resazurin MRC-5 SV2 cell viability assay employed. This is the first report related to chemical composition of C. cowellii plant. The research is now focused on determining the secondary metabolites responsible for the antifungal activity of the total extract.
\end{abstract}

Keywords: Coccoloba cowellii; endemic plant; UHPLC-ESI-QTOF-MS; molecular networking; antibacterial

Supplementary Materials: The following are available online at https:/www.mdpi.com/article/10.3390/ecsoc-24-08289/s1.

Institutional Review Board Statement: Not applicable.

Informed Consent Statement: Not applicable.

Data Availability Statement: The molecular networking job can be publicly accessed at https://eur01.safelinks.protection.outlook.com/?url=https $\% 3 \mathrm{~A} \% 2 \mathrm{~F} \% 2 \mathrm{Fgnps}$.ucsd.edu $\% 2$ FProteoS 
AFe\%2Fstatus.jsp\%3Ftask\%3Da2f9e6e25ca64043a36a3d2fb09270c5\&amp;data=04\%7C01\%7Cpaul. cos\%40uantwerpen.be\%7Cd14ffcfa78f14f0a56ae08d8ff55f3ec\%7C792e08fb2d544a8eaf72202548136 ef6\%7C0\%7C0\%7C637540092792211805\%7CUnknown\%7CTWFpbGZsb3d8eyJWIjoiMC4wLjAwM DAiLCJQIjoiV2luMzIiLCJBTiI6Ik1haWwiLCJXVCI6Mn0\%3D\%7C2000\&amp;sdata=eyepBRIMJy0 H8K\%2Fwidu1\%2FpEMx9pFoA5FDASULxai9YA\%3D\&amp;reserved=0). 